\title{
Natriuretic peptide levels: what's on the horizon?
}

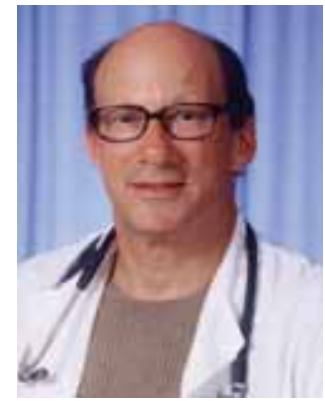

\section{Alan SMaigs}

Veterans Affairs M edical Center, Cardiology 111-A, 3350 La Jolla Village D rive, La Jolla, CA 92161, USA

Tel.: +1 8585528585 ext. 7344 ;

Fax: +1 858522 7490; amaisel@ucsd.edu

\author{
'It is clear that adding [natriuretic \\ peptide] NP levels to clinic al \\ judgment improves the accuracy \\ of diagnosis.'
}

$N$ atriuretic peptide (N P) levels (brain N P [BN P ] and $\mathrm{N}$-terminal prohormone [NTpro]BNP) have taken the cardiology world by storm. The uptake of N Ps have been quicker and more thorough than troponin I and $\mathrm{T}$ - and $\mathrm{C}$-reactive protein. As a result of this excitement, as well as the relatively inexpensive (US\$20) and fast turnaround time (20 $\mathrm{min})$, the possibility of misuse looms large. At the outset, it should be said that N P levels should never be used as a stand-alone test; rather, one that is an adjunct to the patient's history and physical and other laboratory or diagnostic procedures. The two peptides, BN P and $\mathrm{N} T$ proBN $P$, are not interchangeable. While both are of value, one cannot suddenly switch assays and expect the same interpretation of the values. Finally, there is a learning curve for using N P levels.

In the emergency department We know that it is not easy to come up with the correct diagnosis of heart failure $(\mathrm{HF})$ when patients present with shortness of breath. This is because the typical findings of $H F$, such as rales, elevated jugular venous pressure and edema, are not always present. The chest $x$-ray also lacks sensitivity. It is clear that adding N P levels to clinical judgment improves the accuracy of diagnosis [1-3]. Especially important are the rule-out values of less than $100 \mathrm{pg} / \mathrm{ml}$ for BN P and less than $300 \mathrm{pg} / \mathrm{ml}$ for N T proBN P, giving negative predictive values of more than $90 \%$. While N P levels are continuous variables, upper-limit cutpoints are placed for convenience of use. Between the lower limit to rule out $\mathrm{HF}$ and the upper limit to rule in $\mathrm{H} \mathrm{F}$, grey-zone values exist. These are listed in Box 1

Almost two-thirds of patients in the grey zone have the ultimate diagnosis of $\mathrm{HF}$, although their prognosis is much better. $M$ any of these greyzone values occur owing to the release of N PS from the right ventricle. This could be the case in pulmonary hypertension, right ventricular failure or pulmonary embolism. Additionally, the NP level may be related to concomitant diastolic dysfunction or valvular disease. The major caveats in interpreting $\mathrm{N} P$ levels in the emergency department (ED) are renal function and obesity. NP clearance is affected by renal function (N T proBN $P$ is observed to be affected more than $B N P$ ), so values may be slightly higher than normal cutoffs [4]. By contrast, obese patients have lower levels of N P for the severity of $\mathrm{HF}$ than nonobese patients [5].

'The major caveats in interpreting NP levels in the emergency department

(ED) are renal function and obesity.'

Studies have demonstrated that N P use in the ED is cost effective [3,6]. Additionally, it appears that since N P levels are associated with prognosis, they will be effective in the risk stratification of patients. In other words, there are probably cutoffs of N Ps below which a patient with $\mathrm{HF}$ can be treated and sent home, and another cutoff above which patients would be better off being admitted to the hospital for several days.

\section{In the hospital}

$O$ wing to the high readmission rate in hospitalized patients treated for acute decompensation $\mathrm{HF}$, other modalities must be found in order to help solve this problem. N P levels are an evolving, but important, advance in this area. The N P level at the time of admission represents the baseline of euvolemic, that is, 'dry' or 'optivolemic', plus 'wet' N P levels, specifically, the level that is presumed as a result of volume overload. High N P levels in the setting of volume overload almost al ways represent an elevated ventricular end-diastolic pressure [7]. Thus, NP levels may help guide therapy by approaching the dry, baseline level as euvolemia is obtained. I believe N P levels should be considered as a white blood cell count for H F. As treatment progresses, observing the N P level will ensure that the 'infection' (HF) is improving. In particular, BN P has a half-life of only 20 min so levels fall precipitously with beneficial treatment [7]. 
Box 1. Grey-zone natriuretic peptide levels $(\mathrm{pg} / \mathrm{ml})$.

Brain natriuretic peptide

- 100-400

$\mathrm{N}$-terminal prohormone

- $<50$ years old; $300-450$

- 50-75 years; 300-900

- >75 years; 300-1800

Therefore, I obtain N P levels at the time of admission, when there is a change in the clinical state (improving or getting worse) and at the time of discharge. This discharge level should be optimum to follow the patient to help prevent early readmission. The lower the N P level at discharge, the greater the likelihood that the patient will stay out of hospital [8]. In the future, it is likely that therapeutic monitoring with N P levels will become standard therapy.

It also appears likely that, in the future, N P levels will be used in other intensive care unit types of patients. In this setting, N P levels do not necessarily correlate to wedge pressure but, instead, correlate to risk of death and morbidity. Ongoing studies are currently looking at the ability of NP levels to assist in weaning from mechanical ventilation.

\section{Outpatient setting}

The holy grail for N $P$ testing would be to titrate outpatient HF medications ( $\beta$-blockers, angiotensin-converting enzyme inhibitors, etc.) in part by N P levels. Thus, N P levels would be used as surrogates for $\mathrm{HF}$ management. 0 ther types of surrogates have been used to individualize therapy in hypertension, diabetes, lipid disorders and so on. However, there has been no surrogate for $\mathrm{HF}$ that is easy to use, cost effective and altered by effective medications. The N Ps may prove to be valuable in this regard. Studies have been, on the whole, somewhat positive in this regard, but these studies are fraught with issues that still need to be resolved [9]. N P levels might be used to titrate H F medications, but they may also be used to help discern who are the likely candidates for cardiac devices (defibrillators and biventricular pacemakers).

It is clear that patients who suffer decompensation from volume overload on the outside have N P levels well above their baseline. I believe that when symptoms of possible decompensation occur (shortness of breath, edema or weight gain), an N P level of less than $50 \%$ of baseline indicates that decompensation is likely. An increase of $25-50 \%$ is in the gray zone, where clinical judgment is essential. Finally a BNP increase of less than $25 \%$ (or no change) is usually not associated with decompensation [10].

\section{Other areas of interest}

\section{Screening for heart disease}

Studies have demonstrated that, in the right population ( $>1 \%$ prevalence of H F), N P screening prior to echocardiography may be helpful. In this setting, age- and gender-specific cutoffs will be important [11].

\section{Screening potential athletes}

Most children who drop dead while playing sports have underlying disease that might have been detected by N P screening. These include hypertrophic cardiomyopathy, idiopathic cardiomyopathy and right ventricular dysplasia. The fact that N P testing will soon be available by the finger-stick method makes this appealing to those who believe in screening our children.

\section{Diastolic dysfunction}

We are seeing more new-onset $\mathrm{H} F$ patients who have preserved left ventricular dysfunction. Almost all of these patients have elevated N P levels. Thus, many believe that N P levels should be used in the definition of $\mathrm{HF}$ with preserved systolic function.

\section{Altered forms of natriuretic peptides}

It appears that during an episode of decompensation, the wet BNP may not actually be NP, but some altered form. This might include various cleavage products as well as the precursor prohormone.

\section{'In the future, it is likely that thera peutic monitoring with NP levels will become standard therapy.'}

Thisjunk BNP is not physiologically active, but assays are underway that will help evaluate dry versus wet $B N P$, and perhaps contribute to how we monitor and treat acutely decompensated patients.

\section{Acute coronary syndrome}

As N P levels represent ventricular stress, levels often rise in the setting of ischemia. Studies have demonstrated an important independent prognostic value of BNP in the setting of acute coronary syndrome (ACS). In the future, it is likely that NPs will be part of a multimarker panel for risk stratification in ACS. 
Conclusion

NP testing has come a long way and, in my opinion, it will be the benchmark by which other $\mathrm{H} \mathrm{F}$ biomarkers will be tested against in the future. Furthermore, NP levels are not stand-alone tests. U sed in conjunction with other tools that the physician brings to bear, $N \mathrm{P}$ testing has a bright present and an even brighter future.
Financial \& competing interests disclosure

AS M aisel isa consultant for Biosite and has research support from Abbott, Roche and Siemens. The author has no other relevant affiliations or financial involvement with any organization or entity with a financial interest in or financial conflict with the subject matter or materials discussed in the manuscript apart from those di sclosed.

No writing assistance was utilized in the production of this manuscript.
Bibliography

1. M aisel AS, K rishnaswamy $P, N$ owak RM

et al.: Rapid measurement of B-type natriuretic peptide in the emergency diagnosis of heart failure. N . Engl. J. M ed. 347, 161-167 (2002).

2. Januzzi JL Jr, Camargo CA, Anwaruddin S et al.: The $\mathrm{N}$-terminal pro-BN $\mathrm{P}$ investigation of dyspnea in the emergency department (PRIDE) study. Am. J. Cardiol. 95, 948-954 (2005).

3. Mueller $C$, Scholer $A$, Laule-Kilian $K$ et al.: $U$ se of $B$-type natriuretic peptide in the evaluation and management of acute dyspnea. N . Engl. J. M ed. 350, 647-654 (2004).

4. M cCullough PA, D uc P, O mland T et al.: B-type natriuretic peptide and renal function in the diagnosis of heart failure an analysis from breathing not properly (BN P) multinational study. Am. J. Kidney Dis. 41(3), 571-579 (2003).

5. D aniels LB, Clopton $P$, Bhalla $V$ et al.: H ow obesity affects the cut-points for B-type natriuretic peptide in the diagnosis of acute heart failure. Results from the Breathing N ot Properly multinational study. Am. H eart J. 151, 1006-1012 (2006).

6. M oe GW, H owlett J, Januzzi JL, Z owall H : $\mathrm{N}$-terminal pro-B-type natriuretic peptide testing improves the management of patients with suspected acute heart failure: primary results of the $C$ anadian prospective randomized multicenter IM PROVE-CH F study. Circulation 115, 3103-3110 (2007).

7. Kazanegra R, Cheng V, Garcia A et al.: $A$ rapid test for $B$-type natriuretic peptide correlates with falling wedge pressures in patients treated for decompensated heart failure: a pilot study. J. Card. Fail. 7, 21-29 (2001).

8. Cheng V, Kazanagra R, Garcia A et al.: $A$ rapid bedside test for $B$-type peptide predicts treatment outcomes in patients admitted for decompensated heart failure: a pilot study. J. Am. Coll. Cardiol. 37(2), 386-391 (2001).

9. Jourdain $P$, Jondeau $G$, Funck $F$ et al.: Plasma brain natriuretic peptide-guided therapy to improve outcome in heart failure: the STARS-BN P multicenter study. J. Am. Coll. Cardiol. 49, 1733-1739 (2007).

10. $\mathrm{O}$ 'H anlon $\mathrm{R}, \mathrm{O}$ 'Shea $\mathrm{P}$, Ledwidge $\mathrm{M}$ et al.: The biologic variability of B-typenatriuretic peptide and $\mathrm{N}$-terminal pro-B-type natriuretic peptide in stable heart failure patients. J. Card. Fail. 13(1), 50-55 (2007). Erratum: J. Card. Fail. 13(2), 163 (2007).

11. H eidenreich PA, Gubens $M A$, Fonarow $G C$, Konstam M A, Stevenson LW, Shekelle PG: Cost-effectiveness of screening with B-type natriuretic peptide to identify patients with reduced left ventricular ejection fraction. J. Am. Coll. Cardiol. 43, 1019-1026 (2004).

Affiliation

- Alan S M aisel, M D, FACC Veterans Affairs M edical Center, Cardiology 111-A, 3350 La J olla Village D rive, La Jolla, CA 92161, USA

Tel.: +1 8585528585 ext. 7344;

Fax: +1 858522 7490; amaisel@ucsd.edu 